Cultures \& Conflits

08 | hiver 1992

Les conflits après la bipolarité

\title{
Les constitutions dans un monde en proie aux turbulences
}

James Rosenau

\section{(2) OpenEdition \\ 1 Journals}

\section{Édition électronique}

URL : http://journals.openedition.org/conflits/533

DOI : $10.4000 /$ conflits.533

ISSN : $1777-5345$

Éditeur :

CCLS - Centre d'études sur les conflits lilberté et sécurité, L'Harmattan

Édition imprimée

Date de publication : 6 décembre 1992

ISSN : 1157-996X

Référence électronique

James Rosenau, "Les constitutions dans un monde en proie aux turbulences », Cultures \& Conflits [En ligne], 08 | hiver 1992, mis en ligne le 27 janvier 2003, consulté le 30 mars 2021. URL : http://

journals.openedition.org/conflits/533; DOI : https://doi.org/10.4000/conflits.533

Ce document a été généré automatiquement le 30 mars 2021.

Creative Commons License 


\title{
Les constitutions dans un monde en proie aux turbulences
}

\author{
James Rosenau
}

1 Dans un monde "turbulent", fait d'économies chancelantes, de profonds clivages, de vastes transformations internationales et où toutes les formes de pouvoir sont en crise, quels rôles peuvent jouer des réformes constitutionnelles ${ }^{1}$

2 ? Seront-elles capables de contrôler, de s'adapter ou de modifier les formes émergentes de ce qui semble être le "nouvel ordre global" ? Et, si tel est le cas, comment de telles réformes constitutionnelles pourront-elles façonner la dynamique des changements en cours et permettre aux peuples de vivre ensemble, alors qu'il apparait clairement que des tendances centralisatrices, donnent aujourd'hui naissance à des formes transnationales de pouvoir et qu'en même temps d'autres tendances décentralisatrices déplacent la légitimité vers des formes de pouvoir subétatiques ce qui affaiblit du même coup le pouvoir des anciens États-nations?

3 Ces questions sont, sous diverses formes, "à l'ordre du jour", pour tous les types de systèmes : subétatiques, nationaux et internationaux. Tous luttent pour avoir un rôle à leur mesure et pour maintenir et renforcer leur légitimité dans des situations politiques mouvantes et en rapide évolution. Le problème essentiel est de savoir comment établir de nouvelles constructions constitutionnelles, suffisamment fonctionnelles pour emporter l'adhésion de peuples différents, séparés depuis longtemps, habitués à vivre dans des cadres constitutionnels désormais obsolètes. Qu'il s'agisse de la Communauté Européenne testant jusqu'où peut s'imposer une forme transnationale de pouvoir à l'égard des États-nations- ; qu'il s'agisse des Républiques de l'ex-URSS, parfois en cours de désintégration, cherchant, de manière hésitante, à établir un nouveau mode de relations débouchant sur un commonwealth viable ; qu'il s'agisse de la réunification du peuple allemand, que tout le monde souhaitait et que personne n'avait prévue, de celle des deux Corée, ou de la Chine et de Taïwan qui cherchent aussi à se rapprocher; ou encore des Indiens du Canada réclamant leur autonomie. Confédération, fédération, État démocratique, intégration, unification, continentalisme, sécession, autogouvernement, indépendance, nations à systèmes 
multiples, reconnaissance duale... sont autant de concepts devenus partie intégrante des processus politiques à travers lesquels se met en place un nouvel ordre mondial, et sur lesquels politistes et juristes doivent se pencher.

4 Un chercheur en sciences sociales peut avoir deux attitudes quelque peu différentes à l'égard de l'étendue de ces phénomènes constitutionnels; il peut, soit proposer, en fonction de son expérience, un plan d'action en vue de résoudre les différents types de problèmes constitutionnels qui se posent, soit analyser la fertilité du substrat, du terreau, dans lequel ces projets éventuels devront germer. Naturellement, ces deux attitudes doivent être liées, si l'on veut rendre opératoire ces projets. Mais, initialement, ces attitudes font appel à des compétences différentes: un juriste se préoccupe plus de légalité et de justice, tandis qu'un chercheur en sciences sociales se préoccupe surtout des mécanismes souterrains, des changements et des limites effectives au-delà desquels la loi n'opère plus dans une période de transformations profondes. C'est cette dernière approche que nous avons choisi de développer ici.

Rapport entre les faits et les valeurs

5 Quand les experts élaborent une nouvelle construction constitutionnelle - terme que nous utiliserons pour désigner tout projet conçu comme un ensemble de règles permettant la conduite d'une politique au sein d'un système - ou quand ils évaluent les fondements qui doivent leur permettre de se développer, ils se trouvent confrontés à des tensions entre l'analyse des faits et les valeurs qu'ils véhiculent. Les constructions constitutionnelles sont à ce point fondamentales pour le genre de vie et la politique auxquels chacun aspire, que l'on est constamment sujet à de subtiles pressions qui façonnent l'analyse, et ne tiennent pas compte du milieu sociopolitique dans lequel ces propositions seront appliquées. Il est crucial, par exemple, de savoir si c'est un Anglais ou un Français qui évalue et conçoit une construction constitutionnelle destinée à la Communauté Européenne. De fortes tentations inconscientes peuvent vraisemblablement l'entraîner à élaborer un système d'unification favorisant son pays, ce qui gênera du même coup les possibilités qu'un tel projet reçoive une large adhésion au sein de la Communauté.

6 En définitive, la meilleure manière de résoudre ce dilemme entre les valeurs et les faits, est sans doute de reconnaître son existence, de tenir compte des distorsions que nos croyances profondes peuvent entraîner. Mais, reconnaître que nos préférences sousjacentes sont à l'oeuvre n'est pas une mince affaire. Nous sommes toujours tellement persuadés de la justesse et de la valeur des expertises, et des solutions que nous proposons, que cela nous rend vulnérable et risque de nous aveugler sur les cheminements insidieux de nos engagements, compromettant du même coup la rigueur de l'analyse. Il existe pourtant une solution réaliste à ce problème: utiliser nos compétences à l'analyse de situations qui ne touchent pas au domaine de nos convictions profondes. En comparant ainsi un certain nombre de pays confrontés à des problèmes constitutionnels, cela nous entraîne à prendre en compte les divergences, les similitudes et donc la signification de nuances que nous aurions $\mathrm{pu}$ involontairement sous-estimer. Dès lors que notre horizon s'est ainsi élargi, on peut alors étudier un cas plus proche de nous. Pourvu de l'outillage analytique acquis en étudiant un cas lointain, il est peu vraisemblable que notre expertise soit polluée par les valeurs que nous véhiculons. Par exemple, des analystes français ou anglais ayant d'abord enquêté sur les problèmes des deux Corée, seront sans doute mieux placés pour aborder les problèmes de la Communauté Européenne. 
7 Indépendamment des analyses qu'elles génèrent, il y a une vertu heuristique à engager des analyses comparatives et théoriques qui permettent d'élaborer une théorie globale à propos du substrat censé nourrir les constructions constitutionnelles et la probabilité de leur mise en oeuvre pratique. Ceci, pour être sûr que chaque cas a été étudié dans sa spécificité et que les particularismes qui affectent grandement les réformes constitutionnelles possibles seront pris en considération. Toutefois, ce serait mettre la charrue avant les boeufs que de se focaliser sur les particularités spécifiques, avant même de pouvoir saisir les conditions globales, les circonstances générales qui, seules, permettent d'avancer dans la direction désirée.

L'ordre mondial émergent

8 Certains doutent pourtant de la possibilité de faire une analyse globale, compte tenu de la diversité des situations où la nécessité de réformes constitutionnelles se fait sentir. Il est absurde pourrait-on dire, de rechercher un schème interprétatif qui engloberait tous les types de situation : de la sécession à l'unification, des aspirations à l'autonomie au désir d'intégration, des aménagements constitutionnels subétatiques aux accords supranationaux. Et si l'on continue ce raisonnement, il est impossible aussi d'émettre un jugement sur la portée opératoire des constructions constitutionnelles en tant qu'agent causal, pas plus qu'on ne peut les voir sous l'angle d'un produit des réalités sociales économiques et politiques sous-jacentes tant que l'on n'a pas étudié les particularismes de la société à laquelle ces réformes devront s'appliquer.

9 Si la démonstration qui suit ne rejette pas la pertinence de cette argumentation, qui souligne l'historicité des trajectoires spécifiques et des institutions propres à chaque communauté, pays ou région, l'argument qu'une généralisation est imprudente, voire impossible, nous paraît hors de propos et erroné. Nous présumons au contraire que les fondements de l'ordre politique ont des qualités communes invariantes, quel que soit le lieu. Le monde est devenu tellement interdépendant, ses réseaux de communication si étendus, son économie tellement globale, qu'aucune constitution ne saurait être immunisée contre les dynamiques centralisatrices et décentralisatrices à l'origine des crises d'autorité et des processus de restructuration des fondements de la légitimité. Nous retrouverons donc, et c'est notre hypothèse majeure, quel que soit le lieu, les mêmes ingrédients du substrat de l'ordre politique.

10 Dans tous les cas, ce substrat sera constitué d'au moins trois strates différentes, chacune d'entre elles étant nécessaire, mais non suffisante, pour permettre l'enracinement et la maturation éventuelle des réformes constitutionnelles: premièrement une couche profonde constituée par les orientations, les normes et les idées par lesquelles un peuple s'engage dans une adhésion partagée aux règles et aux procédures fondamentales qui rendent un gouvernement des hommes possible; deuxièmement une strate médiane composée par les précédents historiques et les flexibilités institutionnelles qui évitent le rejet des propositions de réformes constitutionnelles; et troisièmement une couche de surface perméable aux circonstances immédiates de l'histoire, par exemple un leader particulièrement créatif, un coup d'État manqué ou une menace externe qui facilite la prise de conscience de la nécessité d'un nouvel ordre global. C'est seulement quand ces trois strates souterraines sont réunies, que l'on peut prévoir la disparition de l'ancien ordre constitutionnel au profit d'un nouveau système constitutionnel cohérent. Il s'ensuit qu'une estimation convaincante des propositions spécifiques qui mèneront à des réformes constitutionnelles dans des pays divisés, des empires éclatés ou des pays régis par 
plusieurs systèmes institutionnels enchevêtrés, nous impose d'étudier en détail le substrat de la politique mondiale afin d'arriver à une compréhension valable des conditions dans lesquelles les édifices constitutionnels s'enracinent ou meurent. Cette tâche "d'extraction" est d'autant plus impérative que le système global connaît une mutation fondamentale: un nouvel ordre mondial émerge. Nous ne faisons pas référence ici à la fin de la Guerre Froide, à la faillite de l'idéologie communiste, au triomphe militaire de la coalition de 32 nations dans la guerre du Golfe, ou aux courants mondiaux de revendication en faveur de nouveaux aménagements constitutionnels. Sans aucun doute, ces nouvelles exigences, la dislocation de l'empire soviétique, les coalitions de l'après-Guerre Froide, se révéleront-elles des sources importantes de changements futurs, mais pour l'instant, de tels événements doivent plutôt être regardés comme les développements de mouvements plus profonds dans les fondations sous-jacentes et apparentes de la politique mondiale. Cette distinction permettra de comprendre le terrain sur lequel on introduit de nouveaux aménagements constitutionnels, en montrant qu'il est nécessaire de les appréhender comme les produits de dynamiques antérieures, favorisées ou freinées par les structures idéelles profondes qui sont la source du comportement politique. En d'autres termes, distinguer les ordres globaux comme l'aboutissement et le développement d'une dynamique antérieure et en tant que fondation sous-jacente, c'est concevoir la montée des revendications pour de nouvelles constructions constitutionnelles dans une perspective élargie. Concevoir ainsi ces revendications incite à rechercher la compréhension du problème au-delà des normes légales et des précédents historiques, dans ces sources comportementales d'où dérive la propension à accepter des réformes constitutionnelles. Les origines et les sources d'un ordre social ne se situent pas dans les chartes, les constitutions, les décrets, ou tout autre texte fondateur, elles naissent dans les esprits et les coeurs d'un peuple, ses habitudes et ses systèmes de croyance. C'est à partir de ces sources qu'un processus à long terme a débuté, processus par lequel les systèmes constitutionnels existants subissent une érosion lente et sans répit.

$11 \mathrm{Au}$ coeur de ce processus à long terme, on trouve une nouvelle construction idéelle qui valorise l'autonomie sur l'allégeance, et l'interdépendance sur l'indépendance. Le terme d'autonomie recouvre une variété de faits et de sens, qui dépendent du contexte dans lequel il est déterminé. Mais, dans tous les cas, le concept d'autonomie implique une propension à contester l'autorité toutes les fois que l'autonomie constitue la seule alternative possible si l'on ne veut pas céder aux traditions et se plier sans réflexion à des mesures non souhaitées, prises par des autorités placées plus haut dans la hiérarchie. Ce principe idéel sous-jacent s'applique à l'individu au sein du groupe, au groupe dans la province, à la province au sein de l'État, à l'État dans l'organisation internationale... La portée du contexte diffère, mais partout on retrouve cet empressement, cette propension à rechercher l'autonomie en fondant la légitimité sur la bonne exécution, le bon fonctionnement, le résultat plutôt que sur l'usage. Mais, aspirer à l'autonomie ne signifie pas forcément rechercher l'indépendance. Une autonomie parfaite, ce serait au contraire la liberté de sélectionner les moyens par lesquels établir l'interdépendance avec les autres individus, groupes, provinces, États et organisations internationales ${ }^{2}$. Notre monde est devenu trop complexe et dynamique pour que l'indépendance puisse à elle seule satisfaire tous nos besoins et nos souhaits. $\mathrm{Au}$ contraire, un large consensus existe au niveau idéel pour reconnaître que besoins et désirs ne peuvent être satisfaits que grâce à des accords de réciprocité, de gré à gré, interdépendance qui n'est pas contradictoire avec le maintien de relations d'autonomie 
au sein d'un système global. Ainsi, les individus concernés ont multiplié les mouvements de contestation difficilement manipulables ; des groupements écologistes ont formé des alliances régionales, les Républiques issues de l'ex-URSS ont cherché à établir un nouveau commonwealth, les États européens concentrent leurs efforts en vue d'établir une union monétaire, les États-Unis et le Canada ont inclus le Mexique dans leur traité de libre-échange.

La transformation des trois paramétres

Si les conséquences majeures de l'émergence d'un ordre global découlent d'une recherche de l'autonomie dans un contexte d'interdépendance, alors que l'ancien ordre s'appuyait quant à lui sur l'allégeance dans un contexte d'indépendance, quelles sont ses structures sous-jacentes? Quelles transformations se sont opérées pour que de nouveaux principes idéels engendrent à leur tour de nouvelles fondations?

La réponse à ces questions intègre les paramètres fondamentaux de la politique mondiale. Si les paramètres d'un système sont conçus comme les frontières au delà desquelles se trouve l'environnement du système - ces facteurs récurrents pouvant avoir un impact sur le système sans être partie intégrante du fonctionnement du système - et dans lequel les variables du système subissent d'incessantes variations, alors il s'en suit que les paramètres sont les caractéristiques fixes d'un système. Ils procurent des lignes de continuité à ce dernier dans la mesure où ils restent constants même lorsque les variables fluctuent. Ils constituent donc les fondements de l'ordre global. Ce sont ces valeurs, prémices, ressources, institutions permanentes qui soustendent et limitent la nature de l'ordre hiérarchique international, qui donnent leur légitimité aux alliances, qui étayent les attitudes à l'égard de la guerre, qui justifient les craintes envers les droits de l'homme, qui façonnent les relations à l'autorité et aux autorités, et ce, malgré et à travers tout ce qui pousse à des variations au sein du système.

14 Si les paramètres de la politique mondiale forment les bases de l'ordre global dominant, un nouvel ordre global ne peut émerger que si ces paramètres subissent une transformation profonde. C'est ce qui est arrivé ces dernières décennies. Pour la première fois, depuis 1648 , date de la signature du traité de Westphalie, les paramètres fondamentaux de la politique mondiale ont subi une altération rapide et étendue, avec pour résultat la pose des fondations d'un nouvel ordre mondial ${ }^{3}$.

Nous avons relevé et identifié un peu partout, trois paramètres essentiels à tout ordre global dominant: une structure d'ensemble de la politique globale (un paramètre macro) ; les structures d'autorité qui relient les macro-collectivités aux citoyens (un paramètre macro-micro) ${ }^{4}$

16 , et les compétences des citoyens (un paramètre micro). Nous pensons que chacun des trois paramètres a subi à notre époque une transformation, et la relative simultanéité de ces transformations semble être la raison majeure de la surprise des commentateurs devant la faillite de l'idéologie communiste en 1989 qui a soudainement rendu visibles les signes de l'émergence d'un nouvel ordre global.

\section{PARAMÈTRE MICRO PARAMÈTRE MACRO-MICRO PARAMÈTRE MACRO}

9 Des individus aux capacités analytiques moindres Des structures de pouvoir qui demeurent tant que les peuples s'appuient sur la tradition et des sources 
constitutionnelles de légitimité pour se plier aux directives émanant des macroinstitutions appropriées Un système anarchique d'États-nations

Des individus aux capacités analytiques supérieures Des structures de pouvoir en crise quand les peuples adoptent un critère performatif de légitimité pour obéir aux directives émanant des macro- institutions Bifurcation d'un système anarchique vers des sous-systèmes multicentrés et étatiques

21 Cette grille résume les changements qui sont intervenus dans les trois paramètres. Mais leur ordre de notation ne doit pas être interprété comme une séquence causale, où les actions des individus précéderaient le comportement des collectivités. Au contraire, enquêter en profondeur sur cet ordre global émergent dépend de la juste appréciation de la nature profondément interactive des trois paramètres et de la reconnaissance du fait que si les individus modèlent les actions et les orientations des collectivités, les politiques, les lois et les buts communs à ces dernières façonnent aussi les individus. Il résulte de cette interaction, un réseau de causalité si imbriqué qu'il est impossible à l'analyse de distinguer précisément et d'isoler les causes des effets. Malgré tout, la rapidité des transformations à l'oeuvre dans la politique mondiale peut être retracée à partir des modifications intervenues au sein de chaque paramètre, qui stimulent et renforcent les transformations des deux autres.

Le paramètre micro : une révolution des compétences et des aptitudes

La transformation de ce paramètre micro réside dans les capacités de mutation et d'adaptation des citoyens partout dans le monde. Les individus sont entrés dans une phase de transformation que l'on peut qualifier de révolution des compétences individuelles. Pour de multiples raisons, qui vont des avancées de la technologie des communications à la complexité croissante de la vie dans un monde toujours plus interdépendant, les individus deviennent de plus en plus aptes à évaluer leur situation dans les affaires internationales et la somme de leurs comportements entraîne des développements politiques globaux et significatifs. On doit inclure parmi ces nouvelles compétences une capacité accrue à faire converger leurs émotions aussi bien qu'à analyser les séquences causales qui sous-tendent et portent le cours des événements politiques.

Ainsi, il serait erroné d'affirmer que la citoyenneté est une forme constante en politique. Le monde ayant rapidement changé et étant devenu plus complexe, cela a eu des conséquences pour les individus englobés par les collectivités qui interagissent sur la scène globale. Certes tant que les individus n'étaient pas impliqués et ne s'investissaient guère dans la vie de la cité, il était justifié de les traiter comme un paramètre constant et de rechercher uniquement au niveau macro les variables expliquant le cours de la politique. Mais aujourd'hui, la révolution des compétences a accru la capacité d'apprentissage des individus, a enrichi leur carte cognitive et a permis l'élaboration de scénarios qui leur permettent d'anticiper le futur. Ce n'est pas une simple coïncidence si récemment les villes du monde entier ont vu des foules immenses réclamer des changements politiques profonds.

La tentation est grande de mesurer l'effet produit par la révolution des compétences en attirant l'attention sur les nombreuses opinions publiques et les foules qui ont protesté contre les régimes autoritaires et réclamé des formes de gouvernement démocratique. Si la dynamique mondiale d'expansion des libertés politiques et la régression du contrôle centralisé des économies sont certainement liés à l'action des citoyens et des opinions publiques qui ont une meilleure appréciation des circonstances et des droits, 
rien ne permet d'affirmer que la révolution des compétences conduira les peuples à la démocratie. Le changement intervenu au niveau du paramètre micro, n'est pas tant celui de ses orientations que celui de son évolution vers de nouvelles capacités à produire des analyses valables. Les peuples, s'ils partagent une plus grande capacité à reconnaître et à articuler leurs valeurs, ne produiront pas et ne partageront pas pour autant des valeurs communes. Ce qui fait que ce changement paramétrique a une portée globale, c'est qu'il permet à des fondamentalistes islamiques, à des paysans asiatiques ou à des citoyens occidentaux de mieux défendre leurs orientations respectives. Cette évolution explique la mondialisation des chocs politiques récents, de Séoul à Prague, de Soweto à Pékin, de Paris aux Territoires occupés, de Belgrade à Rangoon, de la Mongolie à Madagascar... pour ne citer que certains des pays où des actions collectives se sont fait entendre. La transformation de ce paramètre micro est donc devenue évidente : les changements ne se limitent pas à une région particulière du monde.

Il est également important de souligner que la preuve de cette révolution trouve aussi sa source dans la démocratisation de l'éducation, l'usage répandu de la télévision, dans l'utilisation de l'informatique, dans les contacts et les voyages, qui poussent les individus à user de leurs compétences analytiques et émotionnelles. Dans d'autres situations comme les embouteillages en zones urbaines, les pénuries d'eau, les conflits raciaux et les menaces terroristes, les individus sont confrontés à des situations complexes qui les forcent à renoncer à des règles rudimentaires de vie et à élaborer des concepts plus sophistiqués leur permettant de répondre aux défis de la vie quotidienne $^{5}$. Toutefois il ne s'agit pas de dire non plus que tous les individus possèdent, partout, un niveau de compétence et d'aptitude égal. Il est évident qu'une personne issue d'un milieu favorisé aura plus de compétences et d'aptitudes analytiques qu'une personne issue d'un milieu pauvre. Mais, alors que l'espace entre les deux extrémités de ce continuum n'est pas forcément plus étroit que par le passé, l'accroissement des compétences mesurables en chaque point du continuum apparaît suffisant pour contribuer à la transformation de la conduite politique du globe. Plus important encore pour notre propos, le fait est que le nouvel ordre global émergent repose sur des fondations à l'échelle micro, sur des individus qu'on ne peut abuser facilement et qui sont aisément mobilisables sur des objectifs qu'ils comprennent et des moyens qu'ils approuvent ou désapprouvent.

Le paramètre macro-micro : le déplacement de l'autorité

Ce paramètre consiste dans les orientations récurrentes, les pratiques et les modèles qui lient les citoyens (niveau micro) à leurs communautés (niveau macro). En effet, il regroupe des structures de pouvoir et d'autorité par lesquelles de vastes ensembles, étatiques ou groupes privés, mettent en place et maintiennent l'allégeance et la coopération entre leurs membres. Historiquement, ces structures d'autorité se fondent sur des critères traditionnels de légitimité relevant de sources constitutionnelles et légales. Dans un tel environnement, les individus sont habitués à se soumettre aux décisions venant des autorités supérieures. Ils font ce qu'on leur dit de faire, ils obéissent parce que c'est l'usage et que leurs voisins en font autant. La pérennité de ces structures de pouvoir qui demeurent en place pendant des décennies, voire des siècles, n'est donc pas remise en cause; les peuples cédant sans se poser de questions aux "diktats" des gouvernements ou s'effaçant devant le pouvoir décisionnel de leurs propres organisations. Certes, des révolutions ont eu lieu sur le continent américain, en 
France, en Russie et en Chine. Certes, des mouvements de masse ont embrasé l'Europe au milieu du XIXe siècle ${ }^{6}$, mais malgré leur importance historique, aucune de ces révolutions n'a révélé une transformation significative affectant ce paramètre. Partout ces mouvements ont été suivi d'un retour au modèle traditionnel par lequel la souveraineté de l'État et l'autorité gouvernementale forment la pierre de touche de l'ordre politique.

Or, aujourd'hui, la désintégration des pouvoirs ne signifie pas forcément le retour au modèle traditionnel. Au contraire, pour de multiples raisons, dont les capacités d'analyse accrue des citoyens et d'autres facteurs ${ }^{7}$, les fondations de ce paramètre connaissent une érosion souterraine. Partout dans le monde, les sources fondatrices de l'autorité, que ce soit dans des espaces publics ou privés, ont évolué du critère traditionnel vers un critère performatif de la légitimité. Là où les structures d'autorité étaient intactes, elle sont maintenant en crise, la promptitude à l'obéissance des individus dépendant maintenant pour beaucoup de la manière dont ils jugent les performances obtenues par les pouvoirs. Plus le résultat semble approprié, en termes de satisfaction des besoins, de stabilité et de projets d'avenir, plus ils sont prêts à coopérer mais dans le cas contraire, ils sont prêts à user de toutes leurs compétences pour gêner ou compliquer la tâche des autorités.

Conséquence de cette crise générale du pouvoir, les États et les gouvernements deviennent moins efficaces face aux défis auxquels ils se trouvent confrontés et dans la mise en place et la conduite de leurs politiques. Leurs pouvoirs de police leur permettent toujours de maintenir l'ordre mais leur capacité à apporter des réponses autonomes et à résoudre des problèmes d'importance décline car les peuples remettent en cause leur autorité, redéfinissent les fondements de leur légitimité et leur retirent parfois leur assentiment. De tels bouleversements se déroulent maintenant de manière dramatique dans l'ancienne URSS, comme ce fut le cas il y a deux ans dans toute l'Europe de l'Est. Mais cette crise du pouvoir dans les États auparavant communistes n'est que la partie la plus visible de l'iceberg. A l'évidence, cette crise mondiale prend des formes différentes selon les pays et les structures organisationnelles impliquées. Au Canada, la crise des pouvoirs s'enracine dans les conflits linguistiques, culturels et constitutionnels qu'engendre le Québec, à chaque fois que celui-ci semble vouloir faire sécession ou du moins vouloir redéfinir ses relations avec le gouvernement central. En France, la dévolution des pouvoirs a été sanctionnée par une législation nouvelle qui a permis la privatisation et la décentralisation de certaines activités relevant de l'État, les régions recevant des pouvoirs et ayant désormais des attributions qui autrefois relevaient de Paris. En Chine, les provinces ont des attributions plus larges et ignorent ou défient Pékin. En Yougoslavie, la crise a conduit à la violence et à la guerre civile, certaines des composantes de l'État yougoslave, recherchant leur autonomie ou leur indépendance. Dans les pays d'Amérique latine, le défi au pouvoir traditionnel trouve son origine dans les mouvements de guérilla et le trafic de drogue. Ailleurs dans le monde, là où la mutation du critère performatif de légitimité s'est opérée sans provoquer des déplacements d'autorité, aux États-Unis, en Israël, en Argentine, aux Philippines, en Corée du Sud... les impasses l'emportent sur les réussites dans le processus d'invention politique, les gouvernements s'étant révélés incapables de dépasser les divisions sociétales pour entreprendre les actions décisives nécessaires à la résolution de leurs problèmes. 
30 Cette crise globale ne se limite pas aux seuls États et gouvernements. Elle est aussi manifeste dans les juridictions subétatiques, les organisations internationales et les organismes non gouvernementaux ou transnationaux. Dans certains cas, la crise se déploie simultanément à différents niveaux ; ainsi alors que le statut du Québec au sein du Canada était au coeur de la crise, les Indiens Mohawks faisaient pression sur les autorités québécoises pour réclamer une plus grande autonomie. Un phénomène similaire peut s'observer en Moldavie, laquelle a rejeté récemment l'autorité de Moscou, tandis que plusieurs groupes ethniques, russophones et turcs rejetaient l'autorité du nouveau pouvoir moldave. Pareillement, on peut citer d'autres exemples remarquables de crises au sein d'organisations internationales ou transnationales comme l'UNESCO, l'OLP, l'Église catholique romaine et la Mafia, qui, toutes, ont fait l'expérience de dynamiques décentralisatrices, enracinées, au moins en partie, dans la substitution d'un critère de représentativité par un critère performatif de légitimité.

Le déplacement de l'autorité précipité par la crise structurelle des États et des gouvernements au niveau national, opère dans différentes directions. Il dépend pour une bonne part de la perception par les citoyens de la relation entre la finalité des politiques et leurs performances. Dans beaucoup d'instances, cela a entraîné un déplacement de l'autorité "vers le bas", en direction de groupes subétatiques, de minorités ethniques, de pouvoirs locaux, d'organisations à finalité univoque, de regroupements religieux ou linguistiques, de factions politiques, de syndicats... Dans certaines autres instances, ce processus de déplacement de l'autorité s'est effectué "vers le haut", en direction de collectivités englobantes qui transcendent les frontières nationales. Ce déplacement de l'autorité vers le haut s'est produit lorsque ces entités sont devenues les foyers du sentiment de légitimité. Il a bénéficié aux organisations supranationales comme la Communauté Européenne, aux organisations intergouvernementales comme le Comité International de la Croix Rouge, à des organisations non gouvernementales comme Greenpeace, à des organisations professionnelles humanitaires comme Médecins Sans Frontières, à des entreprises multinationales comme IBM, et même à des regroupements sociaux encore balbutiants, qui réunissent des écologistes ou des féministes de différents pays, pour ne rien dire des associations internationales informelles comme celles qui existent dans certaines branches industrielles, des associations formelles, des partis politiques véhiculant une idéologie conservatrice ou socialiste. Il n'est nul besoin d'ajouter que ces déplacements de pouvoirs multidirectionnels renforcent les tensions entre les dynamiques centralisatrices et décentralisatrices qui sous-tendent la turbulence à l'oeuvre dans la politique mondiale.

La remise en cause du principe de souveraineté nationale renforce les crises qui assaillent le paramètre macro-micro. Défier le pouvoir de l'État et diriger les sentiments de légitimité vers des collectivités supranationales ou subétatiques, c'est en effet dénier à l'État la possession du pouvoir ultime de décision, notamment le droit de recourir à la force armée. Or, dans certains cas, le pouvoir s'est structurellement dégradé au point qu'à nombre d'échelons, ses composantes se sont autonomisées tout en ne possédant pas les attributs de la souveraineté. En effet, contrairement à une idée reçue, il n'y a pas de relation directe entre le lieu où s'exerce l'autorité et la souveraineté. Quoi qu'il en soit le trend global du déplacement, de la redistribution de l'autorité contribue à l'érosion du principe de souveraineté. Si un État voit par exemple la mobilisation de ses forces armées contrariées, alors la souveraineté n'est plus le trait 
caractéristique de son existence en tant que collectivité indépendante. Et quand il ne peut prévenir la sécession d'une de ses composantes, l'étendue de sa souveraineté est alors d'autant plus réduite.

Le paramètre macro : une bifurcation des structures globales

Pendant plus de trois siècles, la structure d'ensemble de la politique mondiale aura été fondée sur un système anarchique d'États-nations souverains, qui ne relevaient d'aucune autorité supérieure et réglaient leurs conflits par la négociation ou la guerre. Ces États n'étaient pas les seuls acteurs de la scène politique mondiale mais, traditionnellement ils étaient les collectivités dominantes qui imposaient les règles à suivre. Ce monde centré sur l'Etat (statocentré) a développé sa propre hiérarchie basée sur la manière dont était distribuée la puissance militaire, économique et politique. A des moments historiques différents, en fonction du nombre d'États possédant la plus grande concentration de puissance, l'ensemble du système était dominé par des structures hégémoniques, bipolaires ou multipolaires.

Aujourd'hui, ce monde statocentré n'est plus prédominant. Ceci est dû pour une part à la révolution des compétences, à l'extension des crises d'autorité et à une multiplicité de sources exogènes de turbulences ${ }^{8}$; une bifurcation s'est produite. Un monde multicentré, complexe, constitué d'acteurs relativement autonomes a émergé, avec ses structures, ses processus, et ses propres règles de décision. Les acteurs "libres de souveraineté" de ce monde multicentré sont les entreprises multinationales, les minorités ethniques, les gouvernements locaux, les bureaucraties, les associations professionnelles, les partis politiques et les organisations transnationales... Individuellement, et parfois ensemble, ils entrent en compétition, sont en conflit, coopèrent ou interagissent réciproquement avec les acteurs "contraints par la

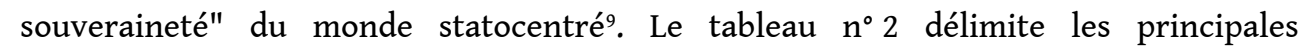
différences de ces deux mondes.

STRUCTURE ET PROCESSUS DANS LES DEUX MONDES DE LA POLITIQUE MONDIALE

\section{MONDE CENTRE SUR L'ÉTAT MONDE MULTICENTRE}

NOMBRE DES ACTEURS INDISPENSABLES Moins de 200 Des centaines de milliers

PRINCIPAL DILEMME DES ACTEURS La sécurité L'autonomie

BUTS PRINCIPAUX DES ACTEURS La préservation de l'intégrité du territoire et la sécurité physique Élargissement des parts du marché mondial. Maintien de l'intégration des sous-systèmes

RECOURS ULTIME POUR REALISER LES OBJECTIFS Le recours à la force armée Refus de coopération ou d'allégeance

PRIORITES NORMATIVES Les processus, particulièrement ceux qui préservent la souveraineté et l'autorité de la loi Les fins, les buts particulièrement ceux qui permettent l'extension des droits humains, de la justice et de la richesse

MODES DE COLLABORATION Par des alliances formelles autant que possible Coalitions temporaires

ÉTENDUE DU PROGRAMME Limitée Illimitée

REGLES D'INTERACTION ENTRE LES ACTEURS Pratiques diplomatiques Réponse "ad hoc", situationnelle 

globale les États, ils n'en sont plus les seuls acteurs clés. Ils doivent faire face à un nouveau défi, affronter des rivaux disparates et résoudre les conflits déclenchés dans leur propre sphère par leurs homologues. L'une des conséquences majeures de la transformation des macro-structures réside évidemment dans la confusion accrue des distributions hiérarchiques qui soutiennent le nouvel ordre global. Les crises d'autorité au sein des États ont non seulement rendu l'ordre international encore plus fluide, mais la bifurcation et l'autonomisation induite des acteurs dans un monde multicentré ont multiplié dans de telles proportions le nombre de ceux qui ont un rôle significatif sur la scène mondiale, que les différences hiérarchiques ont été brouillées au point d'en être devenues méconnaissables et ce bien avant la fin de la guerre froide. Fin qui a malgré tout aggravée la lutte pour la reconnaissance d'un statut international. Ce n'est plus que dans quelques domaines, comme le contrôle des armements ou la question de la prolifération nucléaire, que l'on peut discerner les contours d'une hiérarchie.

Les nouvelles constructions constitutionnelles dans un monde en proie aux turbulences révolution des compétences, le déplacement du pouvoir et la bifurcation des structures globales, quelles opportunités et quelles contraintes affecteront vraisemblablement les processus qui sont l'expression de cette revendication de réformes constitutionnelles? Se révéleront-elles stériles ou productives? Jusqu'à quel point ce qui est à l'oeuvre constitue-t-il le nouveau substrat d'une politique susceptible de donner le jour à de nouveaux ensembles constitutionnels durables et significatifs?

Plusieurs réponses évidentes mais cruciales, peuvent être tirées de la discussion présente. L'une est qu'en dépit des traits exceptionnels qui différencient les situations particulières dont vont dépendre de nouveaux aménagements constitutionnels, tous sont sujets à des stimuli communs qui leur insufflent une similarité sous-jacente et les rendent comparables. Certains possèdent un substrat aux éléments nutritifs plus riches, mais tous tirent leur force ou leur faiblesse du même terreau. Donc, comprendre les difficultés rencontrées par les deux Corée, c'est aussi pouvoir appréhender les problèmes posés par la construction politique de la Communauté Européenne. Plus précisément, partout où des problèmes de réformes constitutionnelles sont apparus, l'enjeu n'est rien moins que l'établissement d'une autorité effective, là où elle s'est effondrée pendant un laps de temps très long et là où il n'y en avait jamais eue 
auparavant. Dans toutes ces situations, la similitude la plus forte découle peut-être de la révolution des compétences qui a contribué à fragiliser les structures d'autorité. Tous ceux qui cherchent à gérer et conduire les affaires publiques, voient depuis longtemps leurs efforts entravés par la pauvreté, le manque de ressources, les conflits internes et d'autres problèmes qui rendent difficiles, le développement de politiques cohérentes et des progrès soutenus vers une vie meilleure. Maintenant, avec l'apparition sur la scène de citoyens toujours plus sensibles à leur bien-être, d'opinions publiques toujours plus rapides à se mobiliser et à descendre dans la rue pour appuyer leurs revendications sous l'oeil de caméras de télévision prêtes à enregistrer l'action du pouvoir, l'autorité des pouvoirs publics devient de plus en plus fragile et sensible à des bouleversements de toute nature. Enfin, quand les citoyens exercent leurs nouvelles compétences à l'occasion de la restructuration des systèmes de gouvernement, des problèmes de territorialité, de l'intégration ou de l'exclusion de la citoyenneté, des procédures de décision, des règles juridiques, bref à l'ensemble des problèmes explosifs que les réformes constitutionnelles cherchent à régler, l'autorité qui s'attache aux institutions gouvernementales semble alors devenir de plus en plus ténue. Comme les constructions constitutionnelles déterminent le degré de contrôle que les individus ont sur leur propre vie et que ces citoyens ont une plus grande possibilité, d'évaluer, d'enquêter et de juger les actes et les réponses que leur offrent leurs dirigeants, jusqu'à un certain point, avec un niveau de liberté qui les décide à agir éventuellement en leur nom propre, ils seront vraisemblablement profondément sensibles à toutes propositions de réformes constitutionnelles qui feront avancer ou compromettront leur bien-être. Que cette sensibilité nouvelle puisse conduire à des crises d'autorité, à placer des gouvernements dans l'impasse, à la paralysie des bureaucraties, n'est pas perçu comme un phénomène plus menaçant que celui des anciennes constitutions qui entravaient les aspirations des citoyens. Seulement les nouvelles constructions constitutionnelles perdent rapidement les bénéfices liés à leur nouveauté, et conservent leurs inconvénients. Ainsi, les crises d'autorité se sont accentuées et les projets de fondation d'États démocratiques, de régimes politiques unifiés, de fédérations d'états ou de toute autre forme de constitution, semblent destinés à sombrer, empêtrés dans un réseau de compétences et de bouleversements tissés par les masses qui disposent soudain de la réalité du pouvoir.

Désormais, même dans les meilleures circonstances, le pouvoir repose sur un réseau précaire de relations au sein duquel celui qui pense et dirige l'ensemble du système, qu'il soit continental ou à l'échelle d'une simple communauté, doit susciter et maintenir l'allégeance des membres que le système englobe. Une autorité effective peut se référer à des textes fondateurs, mais comme nous l'avons déjà signalé, de tels documents ne sont que l'expression écrite de modèles relationnels, des principes idéels et des comportements traditionnels des dirigeants et des peuples. Quand le temps et les circonstances permettent à de tels principes et comportements de s'enraciner, les structures d'autorité qui en résultent sont solides et capables de résister à des chocs sévères. Mais lorsqu'elles sont confrontés à des périodes de transformations profondes et globales, les structures d'autorité les plus solides se révèlent fragiles à l'usage et sujettes à érosion. L'histoire immédiate, nous montre une fois encore qu'un pouvoir solidement implanté bénéficiant d'une longue tradition, peut, une fois remis en cause, s'effondrer à une vitesse vertigineuse, dès que les citoyens s'aperçoivent que le défi (et non plus l'allégeance) est la réponse la plus appropriée à ce pouvoir. La transformation du paramètre macro-micro, et tout particulièrement le remplacement des habitudes 
traditionnelles d'allégeance par un critère performatif de légitimité en tant que fondement des relations avec le pouvoir semble ainsi jouer le rôle d'une contrainte constante à l'égard de la nécessité de nouvelles constructions constitutionnelles. Indépendamment de leur nature, leur réussite dépend de la construction de comportements adéquats qui permettent au citoyen d'accepter et de prendre en compte les nouvelles autorités : une nécessité qui devient hautement aléatoire dans des situations fluides où les individus sont fortement incités à remettre en question le pouvoir plutôt que de s'y soumettre. Si les États totalitaires de l'Europe de l'Est n'ont pu maintenir leur pouvoir effectif, il est difficile d'imaginer que leurs successeurs immédiats pourront rapidement favoriser et stimuler l'apparition d'habitudes d'allégeances à leur égard qui permettraient de fournir un cadre à la mise en oeuvre de leur politique. Les habitudes de leurs citoyens se distinguent par leur caractère irréfléchi, et cela continuera ainsi jusqu'à ce qu'une ou des expériences répétées n'aient confirmé la validité des structures d'autorité mises en place et la validité de leurs propres comportements, les autorisant ainsi à ne pas reconsidérer leur propre attitude une seconde fois. En l'absence de confirmation par l'expérience, des comportements nouveaux peuvent être remis en cause, voire rapidement balayés par des événements contraires et des informations télévisées relatant la crise du pouvoir dans tel ou tel pays, l'échec d'une politique intérieure...

Dit autrement, tant que les structures d'autorité sont fondées sur des modèles habituels de coopération, il est beaucoup plus difficile de les faire parvenir à maturation et de les consolider, que de les détruire une fois qu'elles sont remises en question. Un ordre global peut avoir besoin de plusieurs siècles pour développer de profondes structures comportementales mais nous savons maintenant que de tels ordres, peuvent aussi se défaire très vite. La raison principale de la rapidité de cet effondrement est qu'une fois atteint le point de rupture les individus qui ont réussi à sortir du paradigme idéel qui maintenait intactes les structures de pouvoir le regardent à partir d'une nouvelle perspective où ces structures n'apparaissent plus aussi contraignantes. Les peuples voient qu'ils ont été des victimes, que leur allégeance a permis des politiques auxquelles ils ne peuvent plus souscrire une fois qu'ils se sont libérés du paradigme qui fondait leur comportement. Il suffit de quelques événements libérateurs pour s'attaquer à la démolition des structures d'autorité - on le voit par exemple avec le coup d'État manqué dans l'ex-URSS - et ce n'est qu'avec des instances nombreuses et un leadership politique satisfaisant et performant, qu'une autorité réelle peut construire les habitudes de coopération, de tolérance et d'allégeance qui sont nécessaires au fonctionnement des nouvelles constitutions. Pour en revenir à un exemple empirique spécifique, il faudra des années et peut-être des décennies aux républiques issues de l'Union soviétique pour mettre en place un ensemble constitutionnel qui permettrait l'équilibre entre une diversité décentralisatrice et un ordre centralisateur, et qui fournirait un cadre légal dans lequel une économie capitaliste pourrait prospérer. En ce qui concerne la Communauté Européenne, le temps nécessaire ne sera vraisemblablement pas moindre, encore que les prodromes d'un comportement d'allégeance à l'échelle du continent aient commencé à faire surface. Le temps est donc essentiel.

On pourrait objecter que ces deux cas présentent des caractéristiques particulières qui rendent compte de la lenteur avec laquelle les nouveaux paramètres légaux vont probablement évoluer, que ni les Soviétiques, ni les Européens ne partagent une culture fondée sur des valeurs communes qui pourraient servir de base à la formation rapide 
de nouveaux systèmes de comportements politiques. Le système soviétique ne se serait maintenu dans les faits, que grâce à la direction autoritaire du Parti communiste. De même, pourrait-on faire remarquer que le système étatique européen, s'il a connu une culture commune, ne l'a connue que pour se voir conduire à deux guerres mondiales, ralentissant de la sorte tout changement visant à sortir de ce système interétatique anarchique et à établir un nouvel ensemble constitutionnel.

61 Mais ce raisonnement qui remet fortement en cause la proposition selon laquelle l'apparition d'habitudes susceptibles de soutenir et de rendre opératoires de nouvelles constructions constitutionnelles requiert beaucoup de temps, est problématique. Premièrement, il ne tient pas compte du fait que malgré le manque de culture commune et le fait que ces systèmes politiques aient été maintenus par la force, les chances de survie sont contre tout nouveau paramètre légal qui se développerait autrement que lentement; de même qu'il oublie que les anciens ensembles constitutionnels n'auraient pas fonctionné aussi longtemps qu'ils l'ont fait, s'il en allait différemment. Enfin, l'argumentation s'écroule quand on l'applique à des nations divisées de force. Les deux Allemagne ont en commun la langue et le partage d'une culture, elles ont vécu une union politique et économique pendant plus d'un demisiècle avant leur partition à la fin de la Seconde Guerre mondiale. Malgré cette communauté de culture, depuis la réunification de 1990, les comportements qui devraient soutenir l'autorité nouvelle ne se sont pas mis en place. A l'Est comme à l'Ouest, des voix se font entendre qui suggèrent que les anciens comportements liés à la partition persistent et inhibent la formation d'orientations nouvelles qui rendraient effectives les relations d'autorité. Pareillement, les exigences et les peurs souvent exprimées par l'une ou l'autre des deux Corée ou des deux Chine, relativement à leur contrepartie historique, semblent être des sources de division plus puissantes que ne sont les dynamiques linguistiques et culturelles que partagent les Chinois ou les Coréens entre eux. En outre, il faudrait signaler que les nouvelles constructions constitutionnelles sont par nature rendues plus facilement acceptables quand ce sont les États qui les recherchent comme alternative à des politiques internes ${ }^{10}$. On devrait donc pouvoir transiger avec le principe de souveraineté quand de nouvelles procédures institutionnelles intégratives supplantent celles qui régnaient dans les relations internationales traditionnelles, mais dans une ère où les turbulences sont globales, de tels compromis ne peuvent être perçus comme aussi menaçants que les nouvelles réformes constitutionnelles qui demandent l'adhésion et la coopération de sousgroupes, parfois mutuellement hostiles au sein des États. L'hostilité entre les sousgroupes est généralement profonde et constamment étayée par de multiples litiges, tandis que l'antipathie qui sépare les États est sujette à un effet de distance et n'est pas soumise à un renforcement continuel résistant à l'érosion temporelle. Pour apprécier pleinement ce fait, il suffit de comparer comment l'hostilité du monde libre envers l'Allemagne et le Japon au cours de la Seconde Guerre mondiale a pu changé dans les quarante dernières années tandis qu'en Yougoslavie, l'animosité opiniâtre et constante des Serbes envers leurs concitoyens de Croatie n'a pas cessé. En d'autres termes, il est plus facile à d'anciens ennemis impliqués dans des guerres d'État à État de repartir sur des bases nouvelles dans un nouveau contexte constitutionnel, que de se réconcilier pour des peuples engagés depuis longtemps dans un conflit domestique. Du coup, il est peu surprenant de constater que la marche de la Communauté Européenne vers une nouvelle construction constitutionnelle est moins tumultueuse et plus prometteuse que les propositions faites en vue de restructurer les relations des sous-groupes qui 
réclament leur autonomie dans l'ex-URSS, aux Indes, en Corée ou dans les deux Chine. De même, le renouvellement du traité de libre-échange avec les États-Unis s'est révélé pour les Canadiens une source moindre de divisions, que la révision des relations du Québec avec les autres provinces canadiennes.

La bifurcation du système global sous-tend un autre ensemble de contraintes courantes, partout où l'on recherche de nouvelles constructions constitutionnelles. L'évolution vers un système multicentré avec une grande diversité d'acteurs autonomes et transnationaux, n'accroît pas seulement la densité et la complexité du cadre dans lequel de tels arrangements sont appelés à se mettre en place, il offre aussi des alternatives en terme de foyer de sens, de loyauté et de légitimité pour les individus et les groupes auxquels on propose des réformes censées les intégrer. Lorsqu'on se propose d'inclure au sein d'une nouvelle juridiction élargie des sous-systèmes séparés, ou de désintégrer l'ensemble d'un système existant en juridictions de moindre importance, la diversité des liens émotionnels, des contrats, des relations coutumières de tous ceux qui sont touchés a une telle portée qu'il faut montrer une extraordinaire créativité politique pour mettre en oeuvre de nouveaux projets juridiques qui conserveraient plutôt qu'ils ne remplaceraient ces liens transnationaux et généreraient donc une adhésion suffisante pour qu'ils soient adoptés durablement. Tous les projets constitutionnels qui naissent en ce moment, devront, pour survivre, subir des découpages, des remodelages qui leur donneront l'apparence d'un gigantesque "Gerrymandering"11 transnational qui, par comparaison, fera passer les politiciens américains pour des amateurs.

63 C'est aussi une autre manière de montrer que la bifurcation de la politique mondiale semble entraver les possibilités d'actions et les compétences des autorités nouvellement créées. L'autonomie des acteurs dans un monde multicentré, milite contre le développement de règles juridiques et de frontières politiques nettement définies. De tels acteurs résisteront, et ils le font déjà, à toute construction constitutionnelle qui empiéterait sur leur autonomie, et leurs nouvelles attributions durement gagnées, engrangées au détriment d'États sur le déclin. Au mieux, les nouveaux pouvoirs, s'ils veulent survivre et créer en leur faveur de nouvelles formes d'adhésion, devront agir avec une grande prudence. Certes, une telle perspective initiale, semble jeter une ombre de réticence et apporter un brin de circonspection qui ne sied guère avec le commencement d'une expérience constitutionnelle à hauts risques. Mais procéder autrement, et s'emparer des pouvoirs qu'une nouvelle constitution semble offrir, risque d'aboutir à une situation comparable à celle qu'on a $\mathrm{pu}$ observer à Beyrouth, durant de nombreuses années, quand chaque tentative d'accord entre les coalitions s'effondrait toutes les fois que l'on tentait d'exercer réellement le pouvoir. 


\section{NOTES}

1. Si tant est qu'elles jouent un rôle. Selon les cas nous avons traduit "constitutionnal arrangements" par réformes constitutionnelles ou constructions constitutionnelles. 2. Pour l'élaboration de ce concept d'autonomie, voir James N. Rosenau, "The New Global Order : Underpinnings and Outcomes", article présenté au XVème Congrès mondial de l'Association internationale de sciences politique (Buenos-Aires, 24 juillet 1991). Pour une transformation pratique de ce concept en un principe politique (malheureusement quasiment mort-né), voir l'accord sur l'Union signé par Mikhaïl Gorbatchev et les dix républiques soviétiques, pour préserver une union minimale entre les républiques. Ce plan d'urgence garantissait à chaque république le droit de déterminer de manière indépendante la forme de sa participation à l'union. Francis $\mathrm{X}$. Clines, New York Times, 2 septembre 1991, p. 1.

3. L'analyse qui conclut à la transformation profonde, pour la première fois depuis 1648 , des paramètres du système, se trouve dans James N. Rosenau, Turbulence in World Politics : A Theory of Change and Continuity, Princeton : Princeton University Press, 1990, chap. 5.

4. James N. Rosenau, Turbulence in World Politics, op. cit., pp. 10-11. Pour une formulation qui identifie six paramètres, voir Mark W. Zacher, , in James N. Rosenau and Ernst-Otto Czempiel (eds.), Governance Without Government : Order and Change in World Politics, Cambridge : Cambridge University Press, 1992, Chap. 3.

5. Une analyse exhaustive des types de matériaux empiriques indiquant la profondeur de cette révolution des compétences et des aptitudes se trouve dans James N. Rosenau, Turbulence in World Politics, op. cit., chap. 3.

6. Pour une comparaison historique intéressante entre les périodes du milieu du XIXe siècle et la fin du XXe siècle, voir Sidney Tarrow, "the globalisation of conflict : isn't this where we came in ?" article pour la conférence annuelle de l'Association américaine de science politique, Washington, D.C., 31 août 1991.

7. James N. Rosenau, , Comparative Politics, avril 1992.

8. James N. Rosenau, Turbulence in World Politics, op. cit., Chap. 1, 5, 10 et 13.

9. Pour une discussion de la pertinence de l'emploi des termes "sovereignty free", "sovereignty bound" et pour différencier entre les acteurs étatiques et non étatiques, voir James N. Rosenau,Turbulence in World Politics, op. cit., p. 36.

10. Pour une discussion approfondie sur ce point, voir les essais dans International Aspects of Civil Stafe (sous la direction de) James N. Rosenau, Princeton Princeton University Press, 1964.

11. Aux États-Unis, découpage électoral des circonscriptions particulièrement tortueux dont Gerry Mander fut un expert. 


\section{RÉSUMÉS}

Dans un monde qualifié de "turbulent" confronté à des dynamiques centralisatrices et décentralisatrices qui multiplient les tensions politiques, mettent à mal toutes les formes de pouvoirs et remettent finalement en cause la cohérence du cadre étatique, de nouvelles constructions juridiques et notamment peuvent à n'en point douter jouer un rôle important. James Rosenau analyse ces dynamiques et s'interroge sur les capacités des réformes constitutionnelles à façonner la dynamiques des changements en cours pour permettre au peuples de vivre ensemble. Il souligne les intérêts mais également les limites de ces réformes.

In a " turbulent " World centralising and decentralising forces confront each other creating political tensions, causing harm to all forms of power, and ultimately threatening the very foundations of the State. New legal constructions may play an important role in this respect. James Rosenau analyses the forces at work and examines the capacity of constitutional reforms to impart structure to current dynamics in order to help nations to live together. He points out to the advantages and limits of such reforms.

\section{INDEX}

Mots-clés : droit, Etat-nation, Relations Internationales, souveraineté 